\title{
Relationships between radiographic change, pulmonary function, and bronchoalveolar lavage fluid lymphocytes in farmer's lung disease
}

\author{
YVON CORMIER, JACQUES BÉLANGER, ANDRÉ TARDIF, PIERRE LEBLANC, \\ MICHEL LAVIOLETTE
}

From the Centre de Pneumologie, Hôpital Laval, Ste-Foy, Quebec, Canada

ABSTRACT Ninety four dairy farmers were investigated by chest radiography, pulmonary function tests, and bronchoalveolar lavage. They were divided into five groups-1: 11 subjects with acute farmer's lung; 2: 25 subjects with previously diagnosed farmer's lung who had stayed on their farm; 3: 15 farmers with previously diagnosed farmer's lung who had left the farm; 4: 23 precipitin positive symptomless farmers; 5: 20 precipitin negative symptcmless farmers. The study evaluated the relationships between radiographic changes measured with a scoring system derived from the International Labour Office (ILO) classification, the results of pulmonary function tests, and bronchoalveolar lavage fluid. Thirty eight subjects had radiographic evidence of interstitial pulmonary infiltrates. Group 1 had the highest percentage of lymphocytes recovered by bronchoalveolar lavage (mean 66.3 (SD 19.2)). For all subjects carbon monoxide transfer factor (TLCO) and total lung capacity were negatively correlated with radiographic changes $(r=-0.45$ and $-0.30 ; p<0.001$ and $<0.01$ respectively). TLCO was also negatively correlated with radiographic change in group $2(\mathrm{r}=-0.59, \mathrm{p}<0.005)$. The percentage of lavage lymphocytes was correlated with radiographic changes for all subjects $(r=0.36, p<0.001)$, but this correlation was not seen within groups. This study shows good correlation between radiographic abnormalities, pulmonary function changes and the cellular composition of bronchoalveolar lavage fluid.

Farmer's lung is one of the most frequent types of extrinsic allergic alveolitis; its prevalence varies from one country to another, with estimated figures varying from $0.4 \%^{1}$ to $5 \%^{2}$ of the farming population. The disease is the manifestation of an immunological reaction to microorganisms found in mouldy hay. (Micropolyspora faeni, Thermoactinomyces vulgaris, and Aspergillus species). ${ }^{3}$ The acute phase manifests itself as a febrile illness characterised by dry cough, chest tightness and pains, dyspnoea, cyanosis, chills, and moderate fever four to eight hours after exposure to the antigen. ${ }^{45}$

Chest radiographs and pulmonary function tests are useful both at the time of diagnosis and at follow up visits. A diffuse interstitial micronodular infiltrate is usually seen on the chest radiograph. Typically, pulmonary function tests show a restrictive venti-

Address for reprint requests: Dr Y Cormier, Centre de Pneumologie, Hôpital Laval, 2725 Chemin Ste-Foy, Ste-Foy, PQ, Canada GIV 4G5.

Accepted 23 July 1985 latory defect associated with moderate to severe hypoxaemia. ${ }^{6}$ The intensity of the disease may be determined by the severity of any alteration of pulmonary function and by the degree of hypoxaemia. In other interstitial diseases (sarcoidosis, interstitial fibrosis) the International Labour Office (ILO) system has been shown to be useful, yielding results that correlate with alterations in lung function and with pathological findings. ${ }^{7-9}$

Recently another technique, bronchoalveolar lavage, has gained widespread popularity in the diagnosis and follow up of patients with extrinsic allergic $\omega$ alveolitis and other granulomatous diseases. Bronchoalveolar lavage in acute farmer's lung yields fluid 0

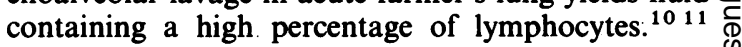
Such a lymphocytic alveolitis is also found in some $\stackrel{\infty}{+}$ symptomless farmers. ${ }^{1213}$ The relationships between 0 chest radiographic changes and the results of pul- $\frac{\vec{D}}{\mathbb{D}}$ monary function tests and of bronchoalveolar lavage $\stackrel{\oplus}{\stackrel{D}{P}}$ have not been clearly established. The purpose of our $\stackrel{\mathbb{Q}}{\Omega}$ study was to evaluate this relationship, using a scoring 
system derived from the ILO classification of radiographs, in a group of subjects with different degrees of disease.

\section{Methods}

Ninety four farmers were included in this study. Eleven had acute farmer's lung at the time of study (group 1). Forty were farmers who had had at least one previous acute episode but were at the time free of farmer's lung symptoms and had not taken corticosteroids for at least three months before the study. Twenty five subjects were still on the farm (group 2), while 15 had ceased all contact with hay (group 3), having left the farm at least two years before this study (range 2-10 years). The diagnosis of farmer's lung in these groups was based on a typical history of dyspnoea, cough, or fever after working with hay, the presence of fine inspiratory rales on physical examination, and a positive result in the precipitin test. ${ }^{14}$ In 15 cases where some doubt persisted, open lung biopsy was undertaken to confirm the diagnosis. Diffuse infiltrates on the chest radiograph and altered pulmonary function supported the diagnosis in most cases.

Group 4 comprised 23 symptomless dairy farmers with precipitins to Micropolyspora faeni, who were randomly selected from the 75 seropositive subjects of our previous epidemiological study. ${ }^{15}$ Group 5 comprised seronegative dairy farmers who were individually matched to subjects of group 4 for age, tobacco consumption, size of farm, and time spent daily in the barn. Three subjects from this group were eliminated: one because he was now precipitin positive and two because we could not complete lavage owing to technical problems. Group 5 therefore finally included 20 subjects. For the precipitin tests Ouchterlony's double diffusion method was used ${ }^{16}$; the result was considered negative if the subject's serum concentrated four times did not react with the antigen.

Subjects from group 1 (acute cases) were usually admitted to hospital and all tests were done within nine days of admission. Subjects from groups 2-5 were seen as outpatients, always in the winter between 1 January and 31 March, when exposure to hay is high. Subjects from groups 2,4 , and 5 came to the hospital after their morning chores on the farm; all had their investigation completed on that day. Pulmonary function tests included measurement of lung volumes - total lung capacity (TLC), residual volume, and functional residual capacity - by the helium dilution method; forced vital capacity (FVC) and forced expiratory volume in one second $\left(\mathrm{FEV}_{1}\right)$ on an Eagle One electronic spirometer (Collins, Braintree, Massachusetts); and carbon monoxide transfer factor (TLCO-that is, diffusing capacity (DLCO)) by the single breath method. ${ }^{17}$ Posteroanterior and lateral chest radiographs were taken. All bronchoalveolar lavages were done in the middle lobe with $300 \mathrm{ml}$ of normal saline (10 aliquots of $30 \mathrm{ml}$ ). Lymphocytes were counted in cytocentrifuged preparations (Cytospin; Shandon, Sewickley, Pennsylvania) stained with Wright-Giemsa and non-specific esterase. As in our previous studies, ${ }^{11}$ a percentage of lymphocytes over $22 \%$ was classified as abnormal and assumed to reflect a current alveolitis.

All 94 chest radiographs were read at the end of the study by one of the investigators (AT), who is an experienced certified B reader; he was unaware of the subject's group or the results of lavage fluid analysis or of pulmonary function tests. Reading of the radiographs was based on the ILO 1979 International Classification of Radiographs of the Pneumoconioses. Radiographs were graded according to the profusion of small opacities; no radiograph in categories $2 / 1$, $2 / 3,3 / 2,3 / 3$, or $3+$ was encountered. We converted the ILO score to an intensity score for easier calculations: $0-, 0 / 0$, and $0 / 1$ were scored as 0 and $1 / 0,1 / 1$, $1 / 2$, and $2 / 2$ as $1,2,3$, and 4 respectively. Each lung was divided into three regions (upper, middle, lower), and an intensity score was assigned to each of the six regions. Thus a score of 0 meant a normal film, and the highest possible score was 24 (diffuse interstitial changes). We refer to this measurement as radiographic score.

For statistical analysis we used analysis of variance and the Student-Newman-Keuls test for multiple comparisons between the five groups. Results are expressed as means (with one standard deviation in parentheses). We calculated the correlation coefficient

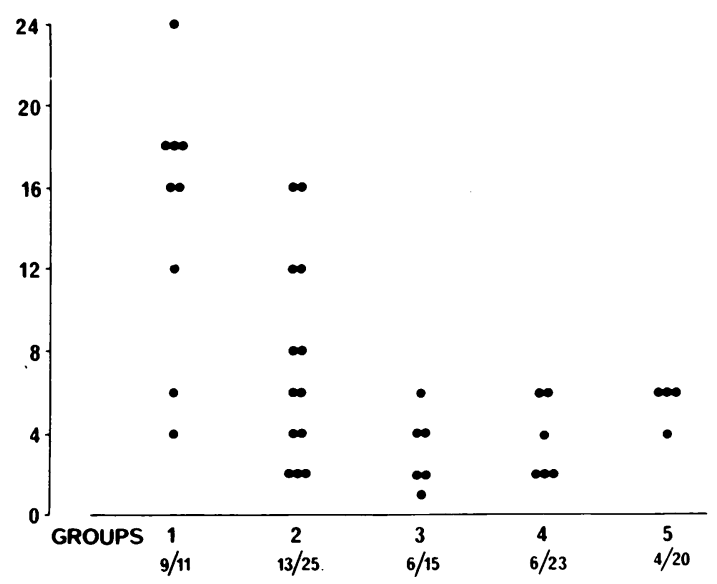

Fig 1 Radiographic scores for the five groups (positive scores only). Ratios below group numbers represent the number of positive films among the total number of subjects in that group. 
TOTAL CELLS $\left(\times 10^{6}\right)$

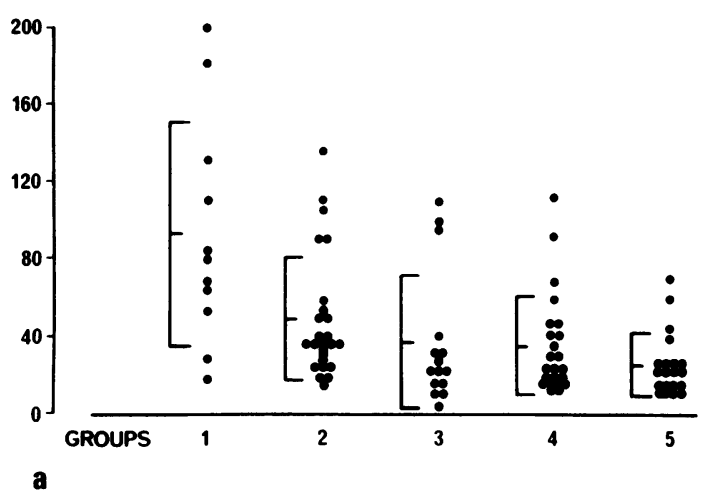

$\%$ OF LYMPHOCYTES

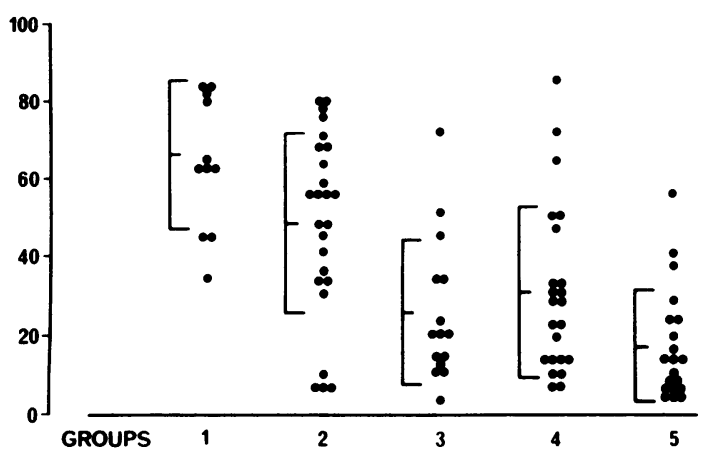

b

Fig 2 (a) Total cells in bronchoalveolar lavage fluid for the five groups, with means and standard deviations. (b) Percentage of lymphocytes in bronchoalveolar lavage fluid.

to assess the correlations between chest radiographic score and the results of bronchoalveolar lavage and tests of pulmonary function.

\section{Results}

Of our 94 farmers, there were 82 men and 12 women with a mean age of 42.6 years (range 18-72). Analysis of variance showed that the groups were of similar age $(p>0.2)$. Four subjects were current cigarette smokers, 17 were ex-smokers, and 73 had never smoked. There were two smokers each in groups 2 and 3; group 3 had more ex-smokers but their mean age when they stopped smoking was relatively young (33.4 years) and therefore should not affect lavage results or other variables. Eighty seven subjects had worked on the farm all their life (including 12 subjects in group 3 who left after their acute disease developed), three subjects had worked there all their life except for one, two, and four years respectively; three subjects had worked on the farm for five to 10 years; and one subject in group 3 was an occasional farm worker at the time of his acute episode. Subjects who had not worked on the farm all their life were evenly distributed between groups.

Nine of 11 subjects of group 1 (acute cases) had a positive radiographic score (fig 1); 19 out of 40 subjects with previous farmer's lung (groups 2 and 3 ) and 10 of the 43 symptomless farmers (groups 4 and 5) also had a positive radiographic score. Thus 38 out of a total of 94 subjects showed some degree of interstitial infiltration on their chest film. The 13 patients with a radiographic score higher than 6 were found in groups 1 and 2.

Patients in groups 1 had a higher total number of cells in their lavage fluid $\left(92.4(58.3) \times 10^{6}\right)$ than subjects in the other groups $(p<0.001$, StudentNewman-Keuls multiple comparisons; fig 2). The mean percentage of lymphocytes was higher in group 1 than in the other groups $(\mathrm{p}<0.001$ compared with groups 3-5; $p<0.05$ compared with group 2). The percentage of lymphocytes in group 2 was also higher than in groups $3-5(p<0.01)$. All patients with acute farmer's lung had a lymphocytic alveolitis with more than $22 \%$ of lymphocytes. The lymphocyte percentage was also increased in 21 out of 25 patients in group 2, six out of 15 subjects in group 3, 14 out of 23 in group 4, and six out of 20 in group 5. We found a positive correlation between the percentage of lymphocytes in the lavage fluid and the radiographic scores for all subjects $(r=0.36, p<0.001$; fig 3$)$. A correlation was not seen, however, when the analysis was made within groups.

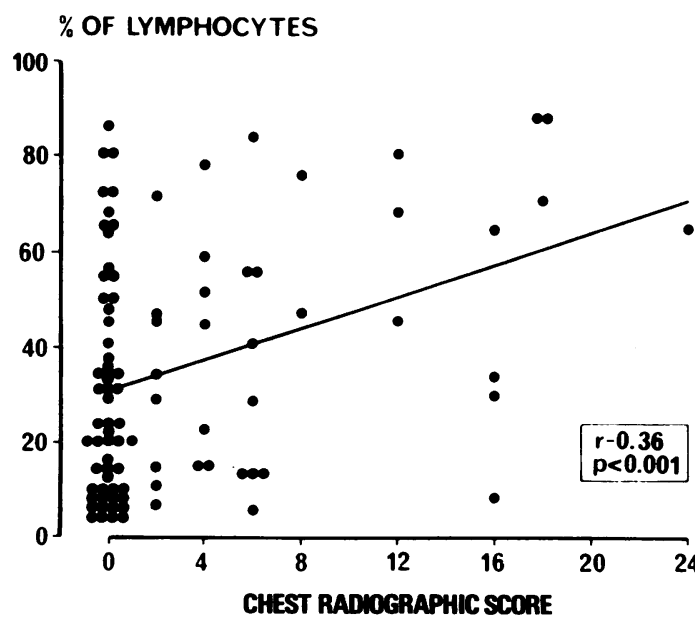

Fig 3 Correlation between the percentage of lymphocytes found in bronchoalveolar lavage fluid and the radiographic score. 


\section{$\%$ OF PREDICTED}

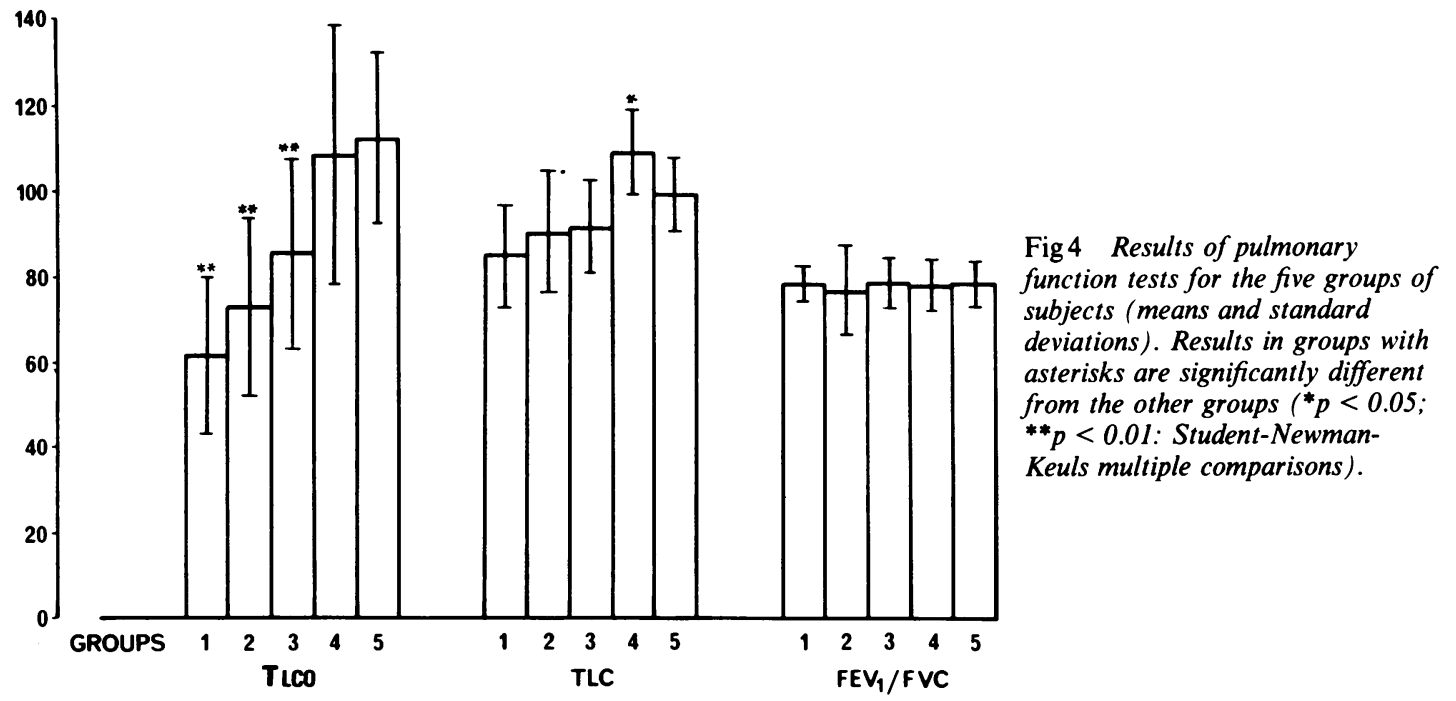

Of the measures of pulmonary function TLco was the most significantly altered, with lower values in groups 1, 2, and 3 than in groups 4 and 5 (p $<0.01$; fig 4). Among the 92 subjects who had TLco measured, a total of 65 had results that were $80 \%$ or more of the normal values predicted and 27 had results less than $80 \%$ of the predicted normal. Of the 27 with abnormal TLCO, 10 were in group 1, 12 in group 2, four in group 3, one in group 4, and none in group 5 (symptomless farmers with negative precipitins). We found a negative correlation between the chest radiographic score and TLCO for all subjects $(r=-0.45$,

TLCO （\% PREDICTED）

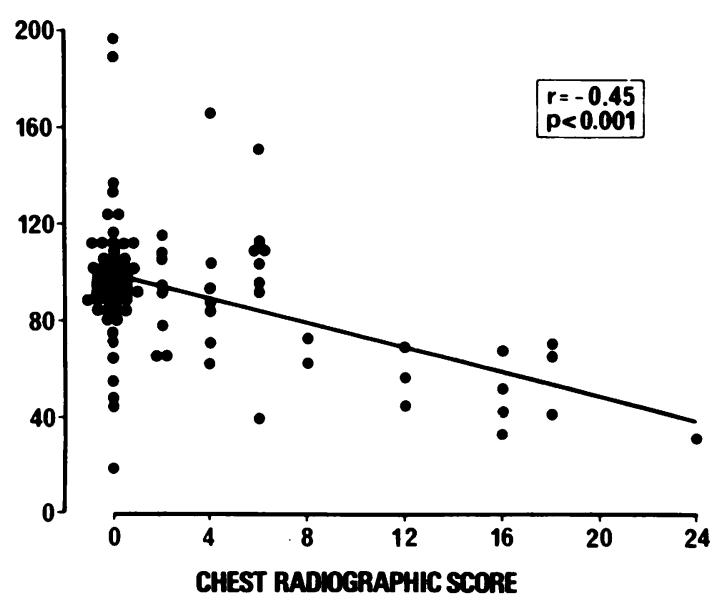

Fig 5 Negative correlation of carbon monoxide transfer factor (TLCO) with radiographic score (all subjects). $\mathrm{p}<0.001$; fig 5). TLco and the radiographic score were also significantly correlated in group 2 patients $(\mathrm{r}=-0.59, \mathrm{p}<0.005)$. Interestingly, all the 13 patients with a radiographic score higher than 6 had a TLCo lower than $80 \%$ of predicted normal.

TLC was measured in 76 of the 94 subjects. Mean values were normal in all five groups, group 4 (symptomless farmers with positive precipitins) having a higher mean ( $p<0.05$; fig 4$)$. Ten out of 76 had a

\section{TLC ( $\%$ OF PREDICTED)}

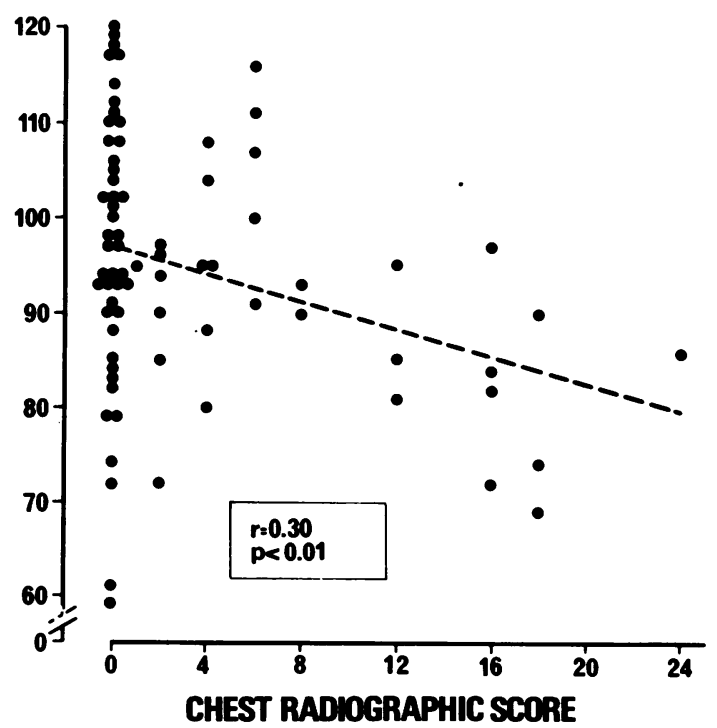

Fig 6 Negative correlation of total lung capacity (TLC) with radiographic score in the 76 subjects who had the test. 
TLC lower than $80 \%$ of the predicted value; four were in group 1, four in group 2, and one each in groups 3 and 5. Like TLCo, TLC was negatively correlated with the radiographic score $(r=-0.30, p<0.01$; fig 6$)$ for all subjects but not within groups.

The mean $\mathrm{FEV}_{1} / \mathrm{FVC}$ was normal for all groups and no significant differences between groups were seen. There was no correlation with the chest radiographic scores.

\section{Discussion}

This study offers a systematic comparison of the relative value of chest radiographs, pulmonary function tests, and bronchoalveolar lavage in a large group of farmers with varied clinical presentations and the data show good correlations between radiographic findings and lung diffusing capacity, total lung capacity, and the percentage of lymphocytes in lavage fluid.

Significant alterations of chest films were associated with a history of farmer's lung: a total of 28 out of 51 subjects in the first three groups $(55 \%)$ had a positive radiographic score. In his long term study Braun found that $39 \%$ of his patients had abnormal radiographs. ${ }^{18}$ Higher scores characterise acute cases of farmer's lung and those with continued contact with hay: all subjects with a radiographic score of more than 6 belonged to groups 1 or 2. No film showed gross changes in parenchymal structure (profusion category of 3 in the ILO classification). To judge from the radiographic appearances none of our patients appeared to suffer from extensive fibrosis; these findings should be interpreted with caution, however, as radiographic changes have often been found to be less striking than the actual clinical picture of farmer's lung, ${ }^{5}$ even if the more severe acute cases do have the greatest changes. ${ }^{19}$

The presence of radiographic abnormalities in 10 out of $\mathbf{4 3}$ symptomless farmers is surprising, five of these having more than $22 \%$ of lymphocytes in their lavage fluid. This alone does not explain the abnormal scores. Barbee also found some degree of radiographic change in six out of 31 symptomless farmers, a proportion similar to that found in our study. ${ }^{20}$ This could be a manifestation of chronic allergic alveolitis, in which acute episodes went undetected. On the other hand, these radiographic findings could reflect shortcomings in the ILO classification for conditions other than pneumoconioses, as has been suggested recently. ${ }^{21}$ Perhaps an investigator who wants to use a scoring system based on the combination of intensity and distribution of abnormal shadows should disregard low scores and consider only greater variations.

Pulmonary function testing may help to complete the picture. TLCO is a good test in farmer's lung. ${ }^{1820}$
In this study 26 of the 27 abnormal TLCo results ( $<80 \%$ of predicted) were found in individuals with acute or chronic farmer's lung. TLCo was negatively correlated with the radiographic score. These two tests seem therefore to be good markers of the presence and intensity of disease, and provide useful clinical tools to support the diagnosis of farmer's lung. Although negatively correlated with the radiographic score, TLC was much less useful than TLCO; only nine out of 50 acute or chronic farmer's lung patients who had it measured had TLC below $80 \%$ of the predicted normal value.

As in a previous study, ${ }^{22}$ there was considerable overlap between the percentages of lymphocytes in lavage fluid in the five groups. Even symptomless dairy farmers had a higher lymphocyte percentage than normal subjects from other studies. ${ }^{2324}$ Although we found a good correlation between radiographic changes and the percentage of lymphyocytes, another study did not find a correlation between these two indices in various types of extrinsic allergic alveolitis. ${ }^{25}$ These discrepancies are probably due to different methods of quantifying radiographic changes. Analysis of bronchoalveolar lavage fluid is a very sensitive diagnostic method; its lack of specificity, however, causes some difficulty in the assessment of the severity and prognosis of acute and chronic farmer's lung. Characterisation of lymphocyte subsets by the use of monoclonal antibodies and analysis of their functional properties by cytotoxicity, chemotactism, and other methods might help to clarify their role in farmer's lung.

This work was supported by the Medical Research Council of Canada and the Association pulmonaire du Québec, and YC is a scholar of the Fonds de Recherche en Santé du Québec.

\section{References}

1 Gruchow HW, Hoffman RG, Marx JJ, Emanuel DA, Rim AA. Precipitating antibodies to farmer's lung antigens in a Wisconsin farming population. Am Rev Respir Dis 1981;124:411-5.

2 Morgan DC, Smyth JT, Lister RW, et al. Chest symptoms in farming communities with special reference to $\Omega$ farmer's lung. Br J Ind Med 1975;32:228-34.

3 Pepys J, Jenkins PA, Festenstein GN, Gregory PH, ㅇ Lacey ME, Skinner FA. Farmer's lung. Thermophilic $\omega$ actinomycetes as a source of "farmer's lung hay" antigen. Lancet 1963;ii:607-11.

4 Fink JN. Hypersensitivity pneumonitis. In: Kirkpatrick $\mathrm{CH}$, Reynolds HY, eds. Immunologic and infectious reactions in the lung. New York: Marcel Dekker, 1976:

5 Emanuel DA, Wenzel FJ, Bowerman CI, Lawton BR. Farmer's lung. Clinical, pathologic and immunologic study of twenty-four patients. Am J Med 1964;37: $392-401$. 
6 Crystal RG, Gadek JE, Ferrans VJ, Fulmer JD, Line BR, Hunninghake GW. Interstitial lung disease: current concepts of pathogenesis, staging and therapy. Am J Med 1981;70:542-68.

7 Davis GS, Dietrich PA, Graham WGB, Navin MC, Vacek PM. Chest radiograph scoring system for non occupational interstitial lung disease [abstract]. Am Rev Respir Dis 1984;129:A70.

8 McLoud TC, Epler GR, Gaensler EA, Burke GW, Carrington CB. A radiographic classification for sarcoidosis. Physiologic correlation. Invest Radiol 1982;17: 129-38.

9 McLoud TC, Carrington CB, Gaensler EA. Diffuse infiltrative lung disease: a new scheme for description. Radiology 1983;149:353-63.

10 Reynolds HY, Fulmer JD, Kazmierowski JA, Roberts WC, Frank MM, Crystal RG. Analysis of cellular and protein content of bronchoalveolar lavage fluid from patients with idiopathic pulmonary fibrosis and chronic hypersensitivity pneumonitis. J Clin Invest 1977;59: 165-75.

11 Leblanc P, Bélanger J, Laviolette M, Cormier Y. Relationship between continued exposure, alveolitis and the clinical state in farmer's lung disease (FLD) [abstract]. Chest 1983;84:357.

12 Solal-Céligny $\mathrm{Ph}$, Laviolette $\mathrm{M}$, Hébert J, Cormier Y. Immune reactions in the lungs of asymptomatic dairy farmers. Am Rev Respir 1982;126:964-7.

13 Cormier Y, Bélanger J, Beaudoin J, Laviolette M, Beaudoin R, Hébert J. Abnormal bronchoalveolar lavage in asymptomatic dairy farmers. Study of lymphocytes. $\mathrm{Am}$ Rev Respir Dis 1984;130:1046-9.

14 Chmelik F, do Pico G, Reed CE, Dickie H. Farmer's lung. J Allergy Clin Immunol 1974;54:180-8.

15 Cormier Y, Bélanger J, Durand P. Factors influencing the development of serum precipitins to farmer's lung antigen in Québec dairy farmers. Thorax 1985;40:138-42.
16 Ouchterlony O. Diffusion-in-gel method for immunological analysis. In: Kallos $\mathrm{P}$, ed. Progress in allergy Basel: Karger, 1958;5:1-78.

17 Ogilvie CM, Forster RE, Blakemore WS, Morton JW. A standardised breath holding technique for the clinical measurement of the diffusing capacity of the lung for carbon monoxide. $J$ Clin Invest 1957;36:1-17.

18 Braun SR, do Pico GA, Tsiatis A, Horvath E, Dickie HA, Rankin J. Farmer's lung disease: long-term clinical and physiologic outcome. Am Rev Respir Dis 1979;119:185-91.

19 Hapke EJ, Seal RME, Thomas GO, Hayes M, Meek JC. Farmer's lung. A clinical, radiographic, functional and serological correlation of acute and chronic stages. Thorax 1968;23:451-68.

20 Barbee RA, Callies Q, Dickie HA, Rankin J. The longterm prognosis in farmers's lung. Am Rev Respir Dis 1968;97:223-31.

21 Epstein DM, Miller WT, Bresnitz EA, Levine MS, Gefter WB. Application of ILO classification to a population without industrial exposure. Am J Roentgenol 1984;142: 53-8.

22 Cormier Y, Béllanger J, Leblanc P, Laviolette M. Physiological significance of the lymphocytic alveolitis in farmer's lung disease (FLD) [abstract]. Am Rev Respir Dis 1984;129:A72.

23 Laviolette $M$. Variation of lymphocyte percentage in bronchoalveolar lavage (BAL) of normal volunteers [abstract]. Chest 1984;86:299.

24 Low RB, Davis GS, Giancola MS. Biochemical analyses of bronchoalveolar lavage fluids of healthy human volunteers smokers and nonsmokers. Am Rev Respir Dis 1978;118:863-75.

25 Hunninghake GW, Gadek JE, Kawanami O, Ferrans VJ, Crystal RG. Inflammatory and immune processes in the human lung in health and disease: evaluation by bronchoalveolar lavage. Am J Pathol 1979;97:149-206. 